\title{
Probability in physics: stochastic, statistical, quantum
}

\author{
David Wallace
}

August 28, 2012

\begin{abstract}
I review the role of probability in contemporary physics and the origin of probabilistic time asymmetry, beginning with the pre-quantum case (both stochastic mechanics and classical statistical mechanics) but concentrating on quantum theory. I argue that quantum mechanics radically changes the pre-quantum situation and that the philosophical nature of objective probability in physics, and of probabilistic asymmetry in time, is dependent on the correct resolution of the quantum measurement problem.
\end{abstract}

\section{Introduction}

If there are objective probabilities — chances — anywhere in nature, physics seems to be the place to look. Most obviously, quantum mechanics apparently makes irreducible probabilistic claims about atomic transitions, the decay of radioactive particles, and the like. More subtly, classical statistical physics makes extensive use of probability distributions over classical microstates, and these are sometimes claimed to be objective probabilities. ${ }^{1}$

This paper has two halves. In the first half (sections 2-6), I summarise the way in which probability figures into generalised stochastic mechanics (that is, the probabilistic generalisation of deterministic classical mechanics) and into classical statistical mechanics; I discuss the philosophical interpretation of probability in both cases, and consider how the two concepts interact if both sorts of probability are simultaneously in use. I also stress the fact that probabilistic dynamics, under weak assumptions, imply a time asymmetry, and explore what assumptions underpin this asymmetry. My impression is that these two pre-quantum situations are the main situations considered by philosophers of probability, and I hope that these sections of the paper provide some help connecting the philosophical discussion to the physics.

However, in the second half (sections 7-10) I consider how the discussion is changed by quantum mechanics. I argue that the probabilistic assumptions

\footnotetext{
${ }^{1}$ In this volume, the chapters by Frisch, Handfield and Wilson, Schwarz, and Weslake explicitly endorse this claim.
} 
of classical statistical mechanics are radically changed by quantum theory, that the role of probability and the origin of probabilistic time asymmetry in quantum theory is strongly dependent on one's preferred resolution of the quantum measurement problem, and that one resolution (the Everett interpretation, or many-worlds theory) suggests an interpretation of objective probability that has no classical analogue and that arguably improves on the pre-quantum situation.

I have tried to write this paper without requiring any acquaintance with contemporary physics or mathematics (beyond a rudimentary acquaintance with complex numbers). As such, I require the reader to take on trust a significant number of technical claims. For the most part I have not attempted to provide detailed references for the claims I make when they rest on standard physics; interested readers are referred to Wallace (2010b), Wallace (2013), and references therein.

\section{Classical mechanics}

At its most abstract, the classical dynamics of $N$ point particles just consists of a set of rules that determine, for each choice of the positions and velocities of the particles, what the rates of change of those positions and velocities are. By iteratively applying this rule, we can determine, for any initial choice of positions and velocities, what the positions and velocities are at all future times. Abstractly, the set of all possible positions and velocities is called the state space of the theory: the dynamics determine, through each point in state space, a unique trajectory for all future times starting at that point. And in fact, the rule can readily be reversed, so that each point determines a unique trajectory for all past times ending at that point. If we call the space of trajectories through state space - that is, the space of functions from possible times into state space - the history space of the $N$-point-particle system, the role of the dynamics is to select a unique history through each point.

We can usefully separate this idea of a history into future histories, which start at a given point and continue forward forever, and past histories, which end at a given point and continue backward forever. A history is then just an ordered pair of one past and one future history.

Essentially all the dynamical theories of classical mechanics (whether they describe the physics of point particles, or planets in motion, or rigid bodies in rotation, or continuous fluids, or electric and magnetic fields, or ... ) have this structure: instantaneous states of a system are represented by points in some state space, and possible histories of the system are represented by trajectories in the state space. The dynamics selects, of all the trajectories through a given point, exactly one which is dynamically allowed. Indeed, the framework is abstract enough to apply to virtually any deterministic dynamical system, given a sufficiently abstract concept of state space. ${ }^{2}$

\footnotetext{
${ }^{2}$ The state space of classical physics is traditionally called 'phase space', a term I avoid for simplicity.
} 
Roughly speaking, ${ }^{3}$ we can think of this history space as a subset of the set of all possible worlds, representing all of the worlds where there are (say) $N$ classical point particles and nothing else. The subset of dynamically allowed histories then represents those possible worlds which are physically possible given the particular sort of classical physics under consideration.

The degrees of freedom of a classical dynamical system are, roughly, ${ }^{4}$ the number of distinct coordinates required to specify a point in state space. In the case of $N$ particles, for instance, the system has $6 N$ degrees of freedom: 3 positions, and 3 velocities, for each of the $N$ particles. When a classical dynamical system has a great many degrees of freedom, describing its full dynamics can be impossibly complicated (a macroscopically large object, under the fiction that its atomic constituents are classical, can easily have $10^{27}$ degrees of freedom!) In this situation, some kind of coarse-grained description is necessary, and a useful example of such is the method of macrostates. Here, the state space is partitioned into subsets (the 'macrostates'), so that each point lies in exactly one subset; in general, macrostates are chosen so that macroscopically similar states lie in the same macrostate. If (mostly for convenience) we also consider the system's state only at a discrete number of times $t_{1}, \ldots t_{n}$, then a given history of the dynamical system determines, for each time $t_{i}$, a macrostate of the system at that time: that is, to every microscopic history is associated a unique macro-history, a unique sequence of macrostates.

In some special cases, to know what macrostate a system has at time $t_{n+1}$, we need to know only its macrostate at time $t_{n}$. For instance, if (for a system of particles) macrostates are defined so that any two states with the same centreof-mass position and velocity lie in the same macrostate, then each macrostate determines a unique macrohistory. In this case, we can say that the system has autonomous macrodynamics. In general, though, to know a system's future macrohistory requires information about its full present state, not just its present macrostate. In principle we can separate out the idea of a futureautonomous macrodynamics from a past-autonomous macrodynamics: a system has future-autonomous (resp. past-autonomous) macrodynamics if the current macrostate uniquely determines all future (resp. all past) macrostates independently of the current state. But in fact, since classical dynamics is past- and future-deterministic, it isn't possible to have one without the other.

Finally, classical dynamics generally possesses two important symmetries: time translation and time reversal. Time translation symmetry means that the dynamics do not depend explicitly on time: that is, the unique dynamical trajectory through a point $x$ is the same whether we consider $x$ to be the state

\footnotetext{
${ }^{3}$ Only roughly, because (i) depending on one's preferred account of possible worlds, the history space may represent, and not simply be, a subset of the possible worlds; (ii) more interestingly, points in state space are specified by positions and velocities, but at least on standard accounts of velocity, the velocity of a particle over a finite period of time is logically determined by the position of that particle over the same period of time. So only that subset of trajectories in state space for which the time derivative of position equals velocity really represent possible worlds.)

4 'Roughly' because often in classical mechanics we treat a position and its conjugate momentum as, collectively, a single degree of freedom.
} 
of the system at one time or at another. In mathematical terms, if $h$ is the unique history with $h(t)=x$, then the unique history $h^{\prime}$ with $h^{\prime}(t+\Delta t)=$ $x$ is given by $h^{\prime}(t+\Delta t)=h(t)$. Time reversal symmetry is a little harder to define (see Albert (2000, ch.1) for a careful but very accessible discussion; see also discussion by Arntzenius and Greaves (2009), Malament (2004), and Earman (2002)) but roughly speaking, it requires that the dynamical system has qualitatively the same structure in the past as in the future. Normally this is imposed by stipulating some appropriate time reversal map $\tau$ of state space to itself, so that if $h$ is the history through some state $x$, the history $h^{\prime}$ through $\tau x$ is given by $h^{\prime}(t)=\tau h(-t)$. (A more precise definition will not be needed for our purposes.)

\section{The stochastic alternative}

Instead of classical mechanics' determinism, we can imagine a probabilistic mechanics where the current state only probabilistically determines future states: a (future-) stochastic mechanics, to use the technical term. Whereas in a futuredeterministic system each given state determines a unique future history, in a future-stochastic system each state $x$ determines a probability measure over future histories starting at $x$. (That is, roughly speaking, it assigns a probability to each future history starting at $x$ : actually the matter is a little more subtle as there will in general be continuum many such histories.) The concept of indeterminism often popularly associated with quantum mechanics in cases like radioactive decay fits the stochastic-dynamics model: if the decaying nucleus has a mean lifetime of $\tau$, then if $x$ is its state at time 0 then the history where it decays at time $t$ gets probability $\mathrm{e}^{-t / \tau}$. The concept of past-stochastic dynamics is defined in the analogous way.

If we imagine discretising time, so that it makes sense to think of the state that immediately follows a given state, we can think of future-stochastic dynamics as specified by a system of transition probabilities: a given state $x$ determines a transition probability $P_{T}(y \mid x)$ for the next state being $y$ conditional on the current state being $x$. (Again, there are subtleties due to the fact that $\mathcal{S}$ is typically continuous). In the continuous-time case this concept of transition probabilities requires more work to make precise, but qualitatively the same ideas apply; in any case, even in a continuous-time system we can still talk about the transition probability for a system currently in state $x$ to be in state $y$ after some fixed time interval. In the case of the decaying nucleus as popularly understood, for instance, for small times $\delta t$ an undecayed state has transition probability $\simeq \delta t / \tau$ into a decayed state and $1-\delta t / \tau$ into an undecayed state, whereas a decayed state stays decayed with probability 1. (And again, for a past-stochastic system we can define transition probabilities into the past in the analogous way.)

Just as in the deterministic case, it makes sense to say that a given futurestochastic system is invariant under time translation: this is to require that the probability assigned by a state $x$ to each history beginning at $x$ is the same 
whatever the time at which the history starts. Equivalently, it is to require that the transition probabilities do not depend on the time.

Importantly, while past- and future-determinism, and time translation invariance, coexist peacefully, in that one and the same system can (and, in classical mechanics, usually does) have all three properties, past- and futurestochasticism and time-translation invariance are incompatible. More precisely: no future-stochastic system which satisfies time-translation invariance is also describable as a time-translation-invariant past-stochastic system, except in the trivial case where the dynamics is really past- and future-deterministic.

We can see why, informally, by considering the decaying nucleus once again. Given a decayed nucleus, for all we know it may have been decayed for an instant, an hour, or a million years). There is no relevant sense in which its probability of having just decayed can be calculated from its present state alone (given the dynamics we have stipulated for it). A more precise proof, and review of the relevant literature, can be found in Uffink (2010).

A possible world whose physics are given by stochastic dynamics, then, has a direction of time which is fundamental: that is, a direction that is built in to the dynamics themselves. It does not depend on contingent details of the matter distribution in that world. ${ }^{5}$

\section{Classical statistical mechanics}

Stochastic dynamics introduces the idea of probability into a physical theory at the level of the fundamental laws, but there is another way in which probabilistic ideas play a role in dynamics. To see this, we return to the deterministic case: suppose we are considering some classical-mechanical system, and that at some initial time $t_{0}$ we specify, not a unique state of the classical system but a probability distribution $\rho\left(t_{0}\right)$ over such states; in mathematical terms, this is to suppose that at $t_{0}$ we specify not a point in the state space but a probability measure over the state space. The interpretation of that probability measure is a vexed question to which I will return but for the moment it may help to conceptualise it simply as a measure of our ignorance of the actual state.

The mathematics of probability, combined with the deterministic dynamics of the system, now determine a unique probability measure over histories: since every point in state space determines a unique history through that point, the probability that history $h$ obtains is just the probability assigned to the state $h\left(t_{0}\right)$ which $h$ assigns to time $t_{0}$. This in turn determines a probability distribution over states at all other times: that is, we can determine a unique probability distribution $\rho(t)$ over states at time $t$, given the original probability distribution $\rho\left(t_{0}\right)$. In fact, we can write down a dynamical evolution equation for $\rho(t)$, which determines the rate of change of $\rho(t)$ as a function of $\rho(t)$. (In classical mechanics, this equation is called the Liouville equation.) Formally,

\footnotetext{
${ }^{5}$ That is, it does not so depend if we take the laws as fixed, and put aside the possibility of a Humean dependence of law on matter of fact. I return to this point later.
} 
this makes the evolution of the probability distribution into just another dynamical system, but where points in the 'state space' are to be understood as probability distributions over physical states of affairs, not as physical states of affairs themselves.

Just as (given a fixed choice of partition of state space into macrostates) an individual dynamical history determines a unique macrohistory (that is: a unique sequence of macrostates), so a probability distribution over histories determines a unique probability distribution over macrohistories. Specifically, the probability of a macrohistory is the total of the probabilities of all of the histories compatible with it.

In the case previously considered, a system had future-autonomous macrodynamics if a given macrostate determined a unique future macrohistory through that state (independent of the actual state). The natural generalisation of this to the statistical case would be to regard a system as having future-autonomous (probabilistic) macrodynamics if a given macrostate determined a unique probability distribution over future macrohistories having that macrostate as their initial history, independent of the actual probability distribution over states. If this were the case, the macrodynamics would constitute a future-stochastic dynamics on the space of macrostates. Since the underlying deterministic dynamics do not depend explicitly on time, that future-stochastic dynamics will be time-translation-invariant. Past-autonomous probabilistic macrodynamics could be defined in the same way.

However, dynamical autonomy in this strong sense is impossible (except in the special case where we have dynamical autonomy in our previous sense, so that all the probabilities are zero or one). The most straightforward way to see this is to note that if the probability distribution is concentrated on a single state (so that that state has probability one and all sets of states not including it have probability zero) then the "probabilistic" macrodynamics collapse back to being deterministic. A subtler reason is that given the time-reversal symmetry of the underlying dynamics, if the system has future-autonomous macrodynamics it also has past-autonomous macrodynamics. But this would mean that we have a dynamics on the space of macrostates which can be described both by a time-translation-invariant future-stochastic dynamics, and by a time-translation-invariant past-stochastic dynamics; we have seen that this is not possible.

However, there are very good (albeit somewhat non-rigorous) grounds to believe that for a certain very wide class of probability distributions (which could be characterised as being all those which do not vary too chaotically and sharply with respect to a certain baseline distribution, the Liouville measure) that the macrodynamics generated by each of these distributions coincide (or very nearly so) and determine a unique probability distribution over future histories for any given macrostate at any time later than the time $t_{0}$ at which the probability distribution is defined. Following Wallace (2010b) and Wallace (2012a, ch.8) I call this class of distributions Simple. If a system does have macrodynamics of this kind, I say that it has almost-future-autonomous macrodynamics: almost future-autonomous because the induced future-stochastic macrodynamics de- 
pend only on some very broad assumptions about the probability distribution, and not on the details of the distribution modulo those assumptions.

Note that the time symmetry of the underlying dynamics means that the time-reversed claim should hold: the macrodynamics determined over past histories (that is, histories ending at time $t_{0}$ ) should be almost-past-autonomous. The reason that this is not contradictory is that the account given violates time translation invariance: in general, distributions that are Simple at time $t_{0}$ are not transformed by the underlying dynamics into Simple distributions in either the future or the past of $t_{0}$. The future evolution of a Simple distribution at a time $t>t_{0}$ has a very complicated microstructure which encodes the fact that the system's past evolution from $t$ to $t_{0}$ is not correctly predicted by the almost-past-autonomous macrodynamics that apply at times before $t_{0}$.

How does this link to real physics? The answer is that the almost-futureautonomous dynamics induced by various forms of deterministic microdynamics turn out to be widely empirically realised in a variety of physical situations, such as the cooling of large objects, or the diffusion of gas into a room, or the stochastic movement of pollen grains (whereas the almost-past-autonomous dynamics are never empirically realised at all). The empirical success of those dynamics could then be explained (under the fiction that the true microdynamics of the Universe is classical) by stipulating that the initial state of the system in question - that is, of the Universe as a whole, if that is the system we wish to study - is determined probabilistically using a Simple probability distribution (which specific distribution is of no further import as far as the dynamics are concerned). We impose this probabilistic boundary condition at the beginning of time (or at least, at the beginning of whatever period of time we can empirically access) because this is the only way to rule out the time-reversed macrodynamics that would otherwise occur to the past of whatever time we choose to impose the boundary condition. This use of a probabilistic boundary condition to explain the asymmetries of observed laws in the classical context is discussed in this volume by Loewer and Albert, and also in, e. g., Albert (2000); for a more detailed version of my own account, see Wallace (2010b). ${ }^{6}$

For completeness - and to link my account more firmly to the philosophy of statistical mechanics literature - I should note that in many cases, including most of those where there are truly macroscopic numbers of degrees of freedom, the induced stochastic macrodynamics is so overwhelmingly concentrated on one macrohistory as to be deterministic to a very high degree of accuracy; in this case, the indeterminism of the macrodynamics has a subtler character. In this case also, we can divide the individual initial states in the first macrostate of a given history into two categories: those that do, and those that do not, deterministically generate the overwhelmingly-most-probable macrohistory. The latter states - call them the thermodynamically Anomalous states - must, by construction, collectively receive overwhelmingly small probability according

\footnotetext{
${ }^{6}$ Albert's condition is only partly probabilistic, and also includes a constraint on the initial macrostate: the 'Low Entropy Past Hypothesis'; in Wallace (2010b) I make the case that this second condition is not needed, at least if the goal is to explain the asymmetry of the observed macroscopic laws.
} 
to any Simple probability measure. The reader should resist the temptation, though, to replace our probabilistic boundary condition with a categorical condition that the initial state is not Anomalous: we have no non-probabilistic characterisation of the Anomalous states, save the circular characterisation that they do not lead to the desired macrohistory.

In any case, the crucial point is that stochastic dynamics that arise from classical mechanics have a directedness that derives not from a fundamental directedness in the dynamics, but from a probabilistically-stated boundary condition. It might be reasonable to call this sort of directedness emergent, rather than fundamental.

\section{$5 \quad$ Interpreting probability in stochastic and sta- tistical mechanics}

My account so far has dealt with (fairly) uncontroversial physics. But the obvious philosophical question to ask is: how are the probability measures to be understood in either stochastic dynamics or statistical mechanics?

There are a great many ways of classifying various sorts of probability, but for my purposes the following will be useful and (I hope) not too oversimplified. A given sort of probability might be (here I basically follow Lewis (1980)):

Credence: A quantification of a rational agent's degree of belief in a hypothesis whose actual truth is unknown (whether the value of that degree of belief is constrained only by the probability calculus, or by other principles of rationality).

Analysed chance: An objective feature of the world, to be analysed in terms of some kind of categorical or at least non-probabilistic feature of the world.

Primitive chance: An objective feature of the world, irreducible to other features.

In the case of stochastic dynamics, it seems difficult at best to understand the probabilities as credences: the transition probability for radioactive decay, for instance, at least seems to be a thoroughly objective feature of the world, to be determined by measurement rather than rational cogitation. (Indeed, it was precisely cases like this that motivated Lewis to argue that even subjectivists about probability required a notion of objective chance.)

If the probabilities of stochastic dynamics are chances, could they be analysed chances? On the face of it, this looks rather difficult: mathematically, the probabilities are represented as a measure over a set of possible histories of the world, but only one element in that set is actual. And recovering this function over all histories from that single history looks logically impossible.

However, the law of large numbers tells us that if a sufficiently large number of independent probabilistic events occur, the relative frequency of any given 
outcome will with high probability be close to the actual probability of that outcome. So for a sufficiently complicated stochastic system, it seems at least plausible that the actual world will with very high probability have a pattern of frequencies from which the probability distribution over all worlds can be recovered.

This strategy of recovering probabilities from long-run relative frequencies is the dominant proposed strategy for analysing chance. Probably its most sophisticated form is the Humean 'best systems analysis' of laws proposed by Lewis $(1986,1994)$ and developed by, e.g., (Loewer 2002; Hoefer 2007), according to which the laws of nature are those hopefully-unique descriptions of the categorical physical world which draw the best balance between simplicity and strength, and which (in particular) might resort to probabilistic language to best explain the relative frequencies of outcomes, without identifying probabilities with those relative frequencies. The task that must be overcome by proponents of such a strategy has two parts:

1. The technical task: actually provide an algorithm to recover, from the categorical facts about the world, the desired probability measure over all worlds (more accurately, over all histories of the dynamical system).

2. The conceptual task: having accomplished the technical task, justify why the recovered measure actually is chance, rather than just being having the formal properties of a probability measure.

The technical task is relatively well defined, the conceptual task less so: just what, if anything, is required to justify such an identification? But one apparently clear aspect of the task is to establish the links between chance and rational inference and decision-making. In particular, we want to understand why it is rational to regard short-run relative frequencies as providing information about chance and why it is rational to regard information about chances as relevant to our actions. Papineau (1996) calls these two links the Inferential and Decision-Theoretic links, respectively; they can also be analysed, following Lewis (1980), as aspects of a general link between credence and chance: the 'Principal Principle. ${ }^{7}$

It lies beyond the scope of this paper to review progress at either task, but it is, I think, fair to say that neither has been completed to anything like general satisfaction, and that there is widespread scepticism as to whether either could be completed. In this context the possibility of taking stochastic probabilities to be primitive chances (and so accomplishing both tasks by postulate) starts to look attractive; Russell's oft-quoted line about the virtues of theft over honest toil does come to mind, though.

What of the probabilities of statistical mechanics? Here there is a considerable tradition (in physics, associated most often with E.Jaynes ${ }^{8}$ and often found in textbooks) to regard the probability measure over states as somehow a measure of ignorance. This strategy can appear attractive at first sight in

\footnotetext{
${ }^{7}$ See also Schwarz, this volume.

${ }^{8}$ See, e. g. , Jaynes (1957a, 1957b).
} 
part because objective chances seem less obviously appropriate for a deterministic universe and in part because the probability measures used in statistical mechanics tend to have a very simple, natural form and so look as if some kind of a priori principle might be used to justify them. (I set aside another family of reasons - based on alleged deep connections between statistical mechanics and information theory - for reasons of space.)

There is room to be sceptical of such principles in probability on their own terms: in particular, they tend to rely on the 'principle of indifference', which has a dubious philosophical status and which anyway is not easy to apply to a theory where a system might be in continuum many states. But more fundamentally, the probability distribution in statistical mechanics grounds objective features of the world. The emergent almost-autonomous stochastic macrodynamics discussed in the previous section have a definite directedness in time their transition probabilities are future-directed, not past-directed - and that directedness is a direct consequence of the imposition of a Simple probability distribution at the start of the universe rather than its end. If the final probability distribution were Simple, the stochastic macrodynamics would play out in the reverse direction; if neither past nor future probability distribution were Simple, there would be no autonomous macrodynamics at all. The same applies to the thermodynamic aspects of irreversibility: phenomena like the melting of ice or the diffusion of gas have a clear time direction which can be tracked back (at least in part) to the probabilistic boundary conditions. If those boundary conditions are simply a matter of credences, it is difficult to see what objective facts about the world are supposed to ground its objective dynamical asymmetries in time. (See Albert (2000, ch.3) for further, acerbic, remarks along these lines.)

This might suggest that statistical-mechanical probabilities ought to be understood as chances, just as for stochastic probabilities — even though the actual dynamics is always deterministic. One way to visualise this proposal is to think of the dynamics of the theory as containing one single stochastic event, happening at the initial time, which sets the initial conditions. Such a theory sounds rather awkward but could plausibly be understood within the Humean best-systems analysis described earlier (that description of the initial condition which best combines simplicity and strength might be a probabilistic condition) or simply taken as a brute postulate.

However, there is a more important disanalogy between the two cases. The probabilities of stochastic dynamics are directly empirically accessible. The only empirical consequence of the initial probability distribution appears to be that the almost-autonomous stochastic macrodynamics are realised - and those macrodynamics take the same form whatever the initial probability distribution is, provided only that it is Simple. Virtually all the specifics of that distribution are empirically inaccessible. If the initial condition is an objective probability claim, then, it is heavily underdetermined by the empirical data.

It is therefore tempting to look for some way to characterise the initial probability distribution that is less specific. One proposal (advocated by, e.g. , Goldstein (2001) and Lebowitz (2007); see also Frigg (2009) and references 
therein) tries to replace the quantitative concept of probability with a qualitative version: "typicality". Such proposals (which remain under development) trade on the previous section's observation that in the case of thermodynamic processes, the Anomalous states whose future time evolution does not generate the overwhelmingly-most-probable future history have very low probability according to any Simple probability measure. If these states can be characterised somehow as wildly atypical, so that it is reasonable to assume that the initial state is not one of them, then no explicit probability measure is required. (The generalisation to macrodynamical processes that are stochastic is not so clear but presumably appeals to the relative frequency of different outcomes when such processes occur many times.)

However, we have now come full circle, as any suggestion that the typicality of the initial state can be assumed on grounds of general a priori reasonableness runs into the objection that the final state is highly atypical, and that the typicality assumption on the initial state plays an essential role in guaranteeing the accuracy of the observed macrodynamics and grounding their time asymmetry. The only way out of this impasse, so far as I can see, is to abandon any hope of grounding the asymmetry of our experience of time in physical facts and instead justify the imposition of the typicality assumption at early times by appeal to some more metaphysically fundamental asymmetry of time; Maudlin (2007, ch.4) has recently made such a proposal, for instance. Of course, this is grist to the mill of those (such as Maudlin) who have independent metaphysical reasons to think that the directedness of time is not simply grounded in contingent physical fact; conversely, those (such as the author) who are suspicious of any metaphysically primitive notion of a direction of time not grounded are likely to be unhappy with this strategy.

\section{Combining statistical mechanics with stochas- tic dynamics}

I have now discussed two separate strategies by which deterministic classical microphysics can be generalised to include probabilities: either by introducing stochastic dynamics, or by going from ordinary mechanics to statistical mechanics. But the two can be combined: nothing prevents us from beginning with a stochastic microdynamics and considering a probability distribution over the initial conditions for that microdynamics.

Prima facie, this suggests that a stochastic statistical mechanics could have two different, independent mechanisms to determine a direction of time: one at the fundamental level, imposed by the direction of the stochastic dynamics, and one at the emergent level, imposed by the imposition of a Simple probabilistic boundary condition at one or other end of time. Indeed, we seem to confront the heady possibility that these mechanisms, being independent, could point in opposite directions!

We should be suspicious of such suggestions, however. Consider, in particu- 
lar, a dynamical theory where the dynamics is deterministic but interrupted by occasional stochastic fluctuations. For instance (here I borrow an example from Albert (2000)) we could supplement the classical mechanics of macroscopically many point particles by adding a stochastic rule which periodically causes the particles to jump small, random distances. ${ }^{9}$ If the collapse mechanism is appropriately structured (if, to be technical, it is reasonably smooth with respect to the Liouville measure) then this additional random noise will have the effect of smoothing out small-scale variations in a given classical probability distribution. This will tend to cause an arbitrary distribution to tend towards a Simple one. Or, in terms of the thermodynamically Anomalous states, it is highly unlikely that a non-Anomalous state will jump to an Anomalous state, but highly likely that an Anomalous state will jump to a non-Anomalous state. (Again, this does not follow a priori for arbitrary choices of collapse rule, but is likely to do so for physically reasonable ones.)

This has a highly desirable consequence (stressed by Albert): in the presence of this stochastic noise, the need for the probabilistic boundary condition goes away, and the existence of almost-autonomous stochastic macrodynamics follows from the existence of stochastic microdynamics. Issues of making sense of the probabilistic initial condition simply go away, and the emergent arrow of time in statistical physics is derived from a fundamental arrow of time in the underlying dynamics. Of course, the problem of making sense of the probabilities of stochastic physics would still remain.

(I make one remark in passing. On this hybrid account, the probabilities of statistical mechanics become just as objective as the probabilities of stochastic dynamics. This calls into question a widespread view in the philosophy of statistical mechanics (stressed by, for instance, Goldstein (2001) and Albert (2000, ch.3)) that physicists are mistaken to associate the thermodynamic state of a system with a probability distribution. The main argument made for this view is that the thermodynamic properties of a system are objective, and so cannot depend on an agent's knowledge of that system. Just so; but if the probability distributions of statistical mechanics are themselves objective, this is no obstacle to taking those distributions seriously as representatives of a system's thermodynamic state. I pursue this argument no further, however, as in my view it is largely obviated by quantum theory, for reasons I expand upon in section 9.)

\section{Quantum theory}

According to one popular conception of quantum theory, it does indeed consist precisely of classical physics supplemented by small random jumps. ${ }^{10}$ If so,

\footnotetext{
${ }^{9}$ Albert officially has in mind a quantum theory - the "GRW dynamical collapse theory", of which more later, but this really serves as motivation for his example, which formally takes place in the classical regime of point particles.

${ }^{10} \mathrm{I}$ once heard it pointed out that a 'quantum leap', much beloved of advertising copywriters, is a leap by the smallest possible amount and in a random direction.
} 
we would have a rather elegant resolution of at least some of the conceptual problems of statistical mechanics, and a unification of the quantum-mechanical arrow of time (induced by the random-jump rule) with the statistical-mechanical arrow of time. However, the popular conception of quantum theory is badly off the mark, and the real situation will turn out to be much more subtle.

What does quantum theory actually look like as a dynamical system? At first sight, rather like a classical dynamical system: it has a state space (the space of rays in Hilbert space, to be precise ${ }^{11}$ ), and a dynamical equation (the Schrödinger equation) which associates a unique trajectory to each state. But things are more complicated than this suggests, because quantum states have a highly suggestive structure. Specifically, the quantum state space is linear: if $\psi_{A}$ and $\psi_{B}$ are possible states of a system, so is $\alpha \psi_{A}+\beta \psi_{B}$, where $\alpha$ and $\beta$ are arbitrary complex numbers satisfying $|\alpha|^{2}+|\beta|^{2}=1$. In the jargon, the new state is a superposition of $\psi_{A}$ and $\psi_{B}$, and $\alpha$ and $\beta$ are the amplitudes of states $\psi_{A}$ and $\psi_{B}$, respectively, in this superposition.

Linearity per se is not a particularly mysterious feature of a state space: the state space of classical electromagnetic theory is linear. ${ }^{12}$ But in quantum theory, certain states seem to describe systems with definite values of some particular quantity (position, say, or angular momentum), so that superpositions of those states seem to have indefinite values of that quantity - and it is not clear what this means. Furthermore, the dynamics of the Schrödinger equation dictate that this indefiniteness can infect the macroscopic realm. To use Schrödinger's infamous example, if we prepare a particle in a superposition of position $A$ and position $B$, and then we set up a device which measures where the particle is and kills some harmless cat if the result is ' $\mathrm{A}$ ', then the dynamics of quantum theory predict that after the device operates the cat will have a state that is a superposition of alive and dead.

Since cats, and macroscopic objects more generally, do not appear to be in indefinite states, this raises the question of how quantum theory can be so empirically successful. The answer is that when we actually use the theory to make predictions, at the end of the process we apply a rule - the Born rule to translate the state into probabilistic predictions. To illustrate by example, if a system's state is such that it has amplitude $\alpha$ of being in position $X$, the Born rule states that the measurement has a probability $|\alpha|^{2}$ of giving result $X$. In the case of the cat, the rule likewise translates the amplitudes for the cat to be alive or dead into probabilities.

This suggests that amplitudes are in some ill-defined sense 'like' probabilities. Indeed, it is tempting (and a number of physicists have succumbed to the temptation) to interpret quantum states in general as probability distributions rather than as physical states (recall that in section 4 I noted that the dynamics of probability distributions over a classical state space can itself be regarded

\footnotetext{
${ }^{11}$ This is a simplification: as I argue in Wallace (2012a, ch.8) and Wallace (2012c), the state space of quantum theory ought really to be taken to be the space of mixed states.

${ }^{12}$ The linearity in question involves real rather than complex numbers - but 'real' numbers are not less abstract than complex numbers, and in fact there are natural formulations of electromagnetism that use complex numbers.
} 
as a deterministic dynamical system). According to this seductive idea, to say that a cat is in a superposition of alive or dead is not to say anything mystical about it being in an indefinite state of alive-ness, but simply and prosaically to say that it has some probability of being alive and some of being dead.

The lures of this idea must be resisted; at least in this straightforward sense, it is unworkable. At base, the reason is the quantum-mechanical phenomenon of interference: quantum amplitudes, unlike probabilities, can cancel out or reinforce one another, instead of simply adding up. To take a stylised example, a particle currently at position $X$ might have amplitude $+1 / \sqrt{2}$ to be at position $W$ in five seconds' time, and the same amplitude to be at position $Z$; the same particle currently at position $Y$ might have amplitude $+1 / \sqrt{2}$ to go to $Z$ and $-1 / \sqrt{2}$ to go to $\mathrm{Z}$. According to the Born rule, whether the particle is at $X$ or at $Y$, it will have probability $1 / 2$ to be found at $Z$ in five seconds' time, and the same probability to be found at $W$. So if the quantum state is simply probabilistic, any superposition of $X$ and $Y$ likewise is equally likely to move to $Z$ as to $W$.

But - because the Schrödinger equation preserves the linear structure of the state space - if the particle initially has amplitude $1 / \sqrt{2}$ to be at $X$ and the same amplitude to be at $Y$, its amplitude to go to $Z$ is just the weighted sums of the $X \rightarrow Z$ and $Y \rightarrow Z$ amplitudes: that is, $(1 / \sqrt{2})(1 / \sqrt{2}+1 \sqrt{2})$ - which is simply 1 . And its amplitude to go to $\mathrm{W}$ is $(1 / \sqrt{2})(1 / \sqrt{2}-1 \sqrt{2})=0$. This cannot be explained on a straightforwardly probabilistic reading of quantum states.

There are more sophisticated ways to understand the difficulty with a probabilistic reading, and to rule out subtler variants (ranging from the classic Kochen-Specker theorem (Kochen and Specker 1967)) to the recent, celebrated result of Pusey, Barrett, and Rudolph (2011)) but ultimately they turn on the same feature of quantum dynamics: interference rules out a probabilistic interpretation of the amplitudes. Essentially the only way around this problem is to abandon any attempt at a realist understanding of quantum theory and fall back on the idea that the theory is simply a black-box device to make empirical predictions; I will ignore such instrumentalism for the purposes of this paper.

To summarise: the quantum states of systems cannot in general be understood probabilistically because of interference phenomena, but the measurement rules of quantum theory seem to require that we understand the state probabilistically when it comes to macroscopic phenomena like measurement processes or cats. In other words, the quantum state seems to be neither physical nor probabilistic but an inconsistent mixture of the two, with physicists interpreting it one way or the other according to the particular situation. This is one way ${ }^{13}$ to state the infamous quantum measurement problem.

Notwithstanding the conceptual severity of the measurement problem, as a practical matter there is now little difficulty in knowing when to treat the quantum state as physical and when to treat it as probabilistic. It is widely

\footnotetext{
${ }^{13}$ It is an admittedly somewhat heterodox way; I defend it more extensively, and more technically, in Wallace (2011).
} 
accepted that the dynamics of the Schrödinger equation are such that interference effects become negligibly small when superpositions become macroscopic in scale. (Quantifying this is the subject matter of so-called 'decoherence theory'; see Schlosshauer (2007) for details, and Zurek (1991) for a somewhat less technical introduction.) In this situation, there is no inconsistency in applying the probabilistic reading of the quantum state. Put in terms of my previous discussion, quantum theory defines, to very good approximation, a future-stochastic macrodynamics. As in the classical case, the macrostates are not precisely defined (though the form of the stochastic macrodynamics is again fairly robust against variations in the method of definition); unlike the classical case, the macrostates cannot be identified with subsets of individual states (because the states, in this regime, are being interpreted as probability distributions over macrostates). (The formal machinery to realise this macrodynamics, and to determine when it is consistently defined, is known as consistent histories or decoherent histories, and is part of decoherence theory; see Hartle (2010) or Halliwell (2010) for conceptually-motivated introduction, and Wallace (2012a, ch.3) for philosophical discussion.)

\section{Resolving the measurement problem}

In one sense, the macrodynamics defined by quantum theory makes quantum physics rather like classical statistical mechanics: in both cases, there is an underlying dynamics which is deterministic, but under certain assumptions we can define from that underlying dynamics a macrodynamics which is stochastic. But in another sense, the two situations are very disanalogous. In the classical case there is no mystery about the ontology of the theory and the relation between micro and macro: we get from one to another by moving from individual states to probability distributions over states, and the stochastic nature of the macrodynamics is inherited from the probability measure of the initial state. In the quantum case the move is instead made by simply treating the quantum state as a probability measure in that (decoherence-defined) regime where it is mathematically consistent to do so. Given that the quantum state cannot consistently be understood as a probability measure in all cases, the conceptual paradox remains, for all the practical utility of decoherence theory.

There are broadly three strategies for resolving that paradox, at least if we want to hold on to the idea that physical theories ought to be descriptions of the world and not instrumentalist toolkits. Two are modificatory strategies: they involve changing quantum physics in order to produce a more conceptually satisfactory theory. The third is purely interpretative: it leaves the mathematical structure of quantum physics intact and attempts to resolve its conceptual difficulties. All three (for the most part ${ }^{14}$ ) have in common a commitment to

\footnotetext{
${ }^{14} \mathrm{I}$ add this qualification for two reasons. Firstly, some advocates of hidden-variable theories (see, e.g., Goldstein and Teufel (2000)) have speculated that in a future quantum-gravity hidden-variable theory, the quantum state could be understood as time-independent and noncontingent, and could then be understood more as a physical law than a physical state. Sec-
} 
the quantum state as being in some sense a physical state, representing physical properties of the world and not just human ignorance of those properties.

The first modificatory strategy — dynamical collapse - introduces an explicit, stochastic modification to the Schrödinger equation, so that the deterministic evolution of the quantum state is punctuated by stochastic transitions. The intended effect of these transitions is to ensure that in situations where the Schrödinger equation predicts a superposition of macroscopically definite states, with some macrostate $X$ having amplitude $\alpha_{X}$, the new dynamics predicts instead that the state will be in one of those macroscopically definite states, with probability $\left|\alpha_{X}\right|^{2}$ of being in state $X$. A dynamical collapse theory, then, transforms the merely formally forward-stochastic dynamics given by decoherence theory (which, recall, metaphysically remains a deterministic theory) into an actually forward-stochastic dynamics.

The second strategy — hidden variables — leaves the Schrödinger equation intact but expands the concept of state, so that the physical state of the system is given not just by the quantum state but by the quantum state together with a state in some additional state space of 'hidden variables' (in many versions of this strategy, the hidden-variable space is taken to the space of positions of individual particles). In one sense, the term 'hidden' is a misnomer, because the intention of the theory is that macroscopic facts supervene on facts about the hidden-variable part of the state, not the quantum state. ${ }^{15}$ The hidden variables have their own dynamics, which may be deterministic or stochastic (but is normally taken to be deterministic) and which causes the future evolution of the hidden-variable state to depend on both the current hidden-variable state and on the quantum state. In turn, by postulating a probability distribution over the hidden variables (though not over the quantum state) at some initial time, a statistical mechanics for the system as a whole is determined. The intention is that all this construction is done in such a way as to produce, in the hiddenvariable part of the state space, an image of the formally stochastic quantum macrodynamics that is actually forward-stochastic (either at the fundamental level, as a consequence of fundamental stocasticity in the hidden-variable dynamics; or at the effective level, as a result of an appropriately-chosen initial probability distribution over the hidden variables). ${ }^{16}$ This will be guaranteed if (i) the quantum macrostates can be characterised in terms of some dynamical variable (such as position); (ii) the hidden variables can be taken to represent the actual value of that variable; (iii) the dynamics, and the hidden variable initial probability, can be chosen to ensure that the probability of the hidden variables having some value is equal to the probability given for that value by

ondly, there is a research program in foundations of quantum theory (see, e. g., Harrigan and Spekkens (2010) and Spekkens (2007)) that tries to eliminate the quantum state entirely from hidden-variable theories. For reasons of space I omit further discussion of these approaches.

${ }^{15}$ It can be questioned whether this intention can in fact be delivered; see Brown and Wallace (2005) for criticism, and Lewis (2007a) and Valentini (2010) for defence (see also Brown (2010) for a reply to Valentini).

${ }^{16}$ I should acknowledge that this way of setting up the idea of a hidden-variable theory is somewhat heterodox: for reasons that are not fully clear to me, the hidden-variable community seems to make little use of the machinery of decoherence theory. 
the Born rule.

Both dynamical collapse theories and hidden-variable theories have been constructed for the case of nonrelativistic particle mechanics. (The classic examples are the GRWP dynamical-collapse theory, named for Ghirardi, Rimini, Weber, and Pearle, and the de-Broglie-Bohm hidden-variable theory; for reviews, see Bassi and Ghirardi (2003) and Cushing, Fine, and Goldstein (1996), respectively.) At present there is no generally accepted way to extend either strategy to the regime of interacting relativistic particles and fields (which is necessary to incorporate, for instance, the results of quantum electrodynamics and particle physics). ${ }^{17}$ For this reason, while both strategies are popular among philosophers of physics they have received relatively little attention within physics proper.

The main interpretative strategy (given a commitment to realism) is the Everett interpretation, which takes the quantum state to be a directly physical state and does not modify or supplement the dynamics. Since the Schrödinger equation predicts the creation of macroscopic superpositions, the Everett interpretation has to take seriously the idea that macroscopic superpositions are possible states of the universe. Given that at the macroscopic level superpositions do not interfere - that is, they branch off from one another but do not recombine - this means that according to the Everett interpretation, at the macroscopic level physical reality has the structure of many constantlybranching approximately-classical states of affairs, co-existing but not interacting with one another. This motivates the alternative name for the Everett interpretation - the 'many-worlds theory' - but it is important to understand the existence of these worlds is not intended as an additional physical postulate but is supposed to follow from the structure and dynamics of unmodified quantum theory. For an introduction to the Everett interpretation, see Wallace (2012b); for a more detailed account see Wallace (2012a); a variety of views pro and con can be found in Saunders, Barrett, Kent, and Wallace (2010).

The Everett interpretation has the major technical advantage of being applicable to all known quantum theories, not just to the relatively restricted regime of nonrelativistic particle physics; I think it is largely for this reason that it is significantly the most popular of my three strategies among physicists (which is not to say that it is the most popular solution to the measurement problem overall: at least as many physicists are attracted by various more-or-less-operationalist strategies, and of course the great majority adopt the so-called 'shut up and calculate interpretation'!). It is, however, generally regarded amongst philosophers as being more conceptually problematic than the hidden-variable or dynamicalcollapse strategies. The main philosophical concerns raised are (i) what justifies regarding the quantum state as really (albeit emergently) representing multiple branching worlds, and (ii) how probability is to be understood in a situ-

\footnotetext{
${ }^{17}$ This is not to say that there has been no progress on this front. The nearest thing to a dynamical-collapse theory in the relativistic regime is Tumulka's theory (Tumulka 2006), which applies in the case where there are no interactions; relativistic hidden-variable theories have been proposed by Durr et al (2004, 2005), Struyve and Westman (2007), and Colin and Struyve (2007).
} 
ation where all possible outcomes of a quantum measurement are physically realised. The former lies largely beyond the scope of this paper; in my own view, it is unproblematic once we commit to a broadly functionalist account of the relation between higher-order ontology and the underlying micro-ontology, according to which the 'worlds' are higher-level structures instantiated in the microphysics. I expand upon this in Wallace (2003, 2010a) and (Wallace 2012a, ch.2); see Maudlin (2010) and Hawthorne (2010) for dissenting views (and Ladyman (2010) for a reply to Hawthorne). The latter is best considered (for the purposes of this paper) in the wider context of probability in quantum mechanics, and is the topic of the section 10; before that, though, I need to consider the statistical-mechanics version of quantum theory.

\section{Quantum statistical mechanics and the arrow of time in quantum theory}

In section 7 , I claimed that quantum theory leads (at least formally) to a forward-stochastic macrodynamics, due to the operation of decoherence theory. The observant reader will recall that in section 3 I claimed that no nontrivial stochastic process can be simultaneously forwards-stochastic, backwardsstochastic, and time-translation-invariant, so (given time-translation invariance) that stochastic process must be time-asymmetric. This leads to a conceptual problem over and above the quantum measurement problem: where does this time asymmetry come from, given that it is not manifest in the (forwards- and backwards-)deterministic Schrödinger equation?

Almost as a matter of logic, the answer must lie in the initial conditions, and indeed it does. The derivations of emergent forward-stochastic physics that occur in decoherence theory rely on certain assumptions about the initial quantum state: it is assumed not to have too delicate a structure of phases and magnitudes. The nature of this boundary condition is very similar from a mathematical point of view to the Simple condition imposed in classical statistical mechanics upon probabilistic initial conditions, and so again (and again following Wallace 2010b) I call states Simple if they satisfy these assumptions; however, from a conceptual point of view the assumption has a very different character, as it is a constraint not on any probability distribution over initial states but on the actual initial state.

In any case, the direction of time present in (unmodified) quantum mechanics is emergent, just as in classical statistical mechanics: it arises as a consequence of certain boundary conditions.

These structural analogies between quantum mechanics and classical statistical mechanics ought to cause us to be cautious in any assumption that quantum statistical mechanics can be understood by analogy with classical statistical mechanics. Recall that in classical mechanics, we obtain a statistical mechanics from an underlying deterministic dynamics by placing a probability measure over initial conditions; if that probability measure is Simple, we obtain 
a macrodynamics (for later times than the initial condition) that is effectively forward-stochastic and autonomous from the microscopic details. It might seem that the same move is available in quantum theory: by placing a probability measure over deterministically-evolving quantum states, we might expect to obtain a quantum statistical mechanics, and to find emergent stochasticity by constraining that probability distribution to be Simple.

However, we have seen that we obtain emergent stochasticity in quantum theory simply by imposing a non-probabilistic boundary condition on the initial state of a quantum system, if it has macroscopically many degrees of freedom. The imposition of a further probabilistic boundary condition does not seem to give rise to anything qualitatively new, and in fact it is far from clear that it does any quantitative work in the quantum theory of macroscopic systems either. (Certainly, I am not aware of any quantum-mechanical derivation of emergent irreversibility or stochasticity which relies on any such assumption. ${ }^{18}$ )

The point can be put directly as follows. In classical mechanics, we can consider the dynamics of individual systems or of probability distributions over such systems; we can also consider the dynamics of systems with a small number of degrees of freedom, or with macroscopically many degrees of freedom. The dynamics of systems with macroscopically-many degrees of freedom is not classical statistical mechanics: we obtain the conceptual novelties of statistical mechanics only by going from individual states to probability measures over states (albeit we may be able to finesse this move via considerations of typicality or the like) and then imposing certain boundary conditions on those distributions. In quantum mechanics, by contrast, the discipline of quantum statistical mechanics is obtained simply from applying ordinary quantum mechanics to systems with very many degrees of freedom and then imposing certain boundary conditions on individual quantum states.

From a conceptual point of view, this should perhaps not be surprising. I stressed in section 7 that quantum states behave like probability distributions in the macroscopic regime. This suggests that we should think of the classical limit of quantum mechanics as already being classical statistical mechanics (a point that has been stressed by Ballentine $(1970,1990)$ ). If so, the success of classical statistical mechanics gives us no particular reason to make the statistical move in the quantum case.

Indeed, the reverse is true. Insofar as classical mechanics is of interest to us only because it is a good approximation to quantum mechanics in certain situations, the probability distributions in classical statistical mechanics arguably should be understood as classical limits of individual quantum states, not as probability distributions at all ${ }^{19}$ (except to the degree that individual quantum

\footnotetext{
${ }^{18} \mathrm{It}$ is true that many such derivations make use of the machinery of mixed states (aka density operators) and that mixed states do have an interpretation as probabilistic mixtures of pure states. However: (i) in those cases of which I am aware, nothing essential (as opposed to calculational) seems to change if the system's state is instead treated as pure; (ii) it is far from clear that the probabilistic interpretation is correct, given that mixed states also have an interpretation as possible states of individual systems in (at least) those circumstances where the system is entangled with other systems. I expand upon this point in Wallace (2012c).

${ }^{19} \mathrm{It}$ is interesting to note that the main formalism used in physics to study semiclassical
} 
states should be so understood). If so, the conceptual problems of probability in classical statistical mechanics which vexed us in section 5 dissolve entirely: they are artefacts of classical physics, with at most historical significance. There remains a need for a constraint on initial conditions to deliver stochasticity at the macro level, and this constraint may be philosphically problematic, ${ }^{20}$ but it need not be understood as a probabilistic constraint.

\section{Probability in quantum theory and its alter- natives}

If quantum theory dissolves the probabilistic puzzles of classical statistical mechanics, still it generates its own puzzles, which differ across the three strategies to solve the measurement problem (dynamical collapse, hidden variables, Everett) which I reviewed in section 8. The situation is cleanest in dynamicalcollapse theories, which are straightforwardly stochastic theories in the sense of section 3: the discussion of stochastic chance in section 5 applies directly. The state space of the theory is the state space of quantum theory; the dynamics is specified by means of a rule mapping states to probability distribution over future histories in that state space; the actual world consists of just one history in state space; if probability is to be analysed in terms of some non-probabilistic fact, the only candidate appears to be something like relative frequencies within that history; formally defining a probability from information about these relative frequencies is not trivial; even if it could be defined, it is not clear on what grounds it would count as being probability, and in particular, why it should conform to the role played by chance in our inferential and decision-theoretic activities. On the other hand, the stochastic dynamics probably suffices to remove any residual mystery about the condition of Simplicity on initial quantum states required for the derivation of forward-stochasticity at the macro level: it seems plausible (although I am not aware of technical results) that for any reasonable dynamical-collapse rule, the argument of section 6 would go through mutatis mutandis and remove the need for such a condition. (Indeed, dynamical collapse theories are precisely the inspiration for Albert's consideration of stochastic jumps in classical mechanics, as discussed in that section.) The temporal asymmetry in a dynamical-collapse theory would then be a consequence of an asymmetry in the dynamics, and would be independent of any detailed constraints on the initial state.

In a deterministic hidden variable theory such as the de Broglie-Bohm theory, the quantum probabilities arise from a probabilistic constraint on the initial values of the hidden variables; indeed, the constraint is much sharper than in classical statistical mechanics, with the choice of probability distribution being entirely specified by the quantum state if the predictions of quantum theory are

quantum theory - the Wigner function formalism — identifies quantum states with functions on phase space which approximate classical probability distributions obeying the Liouville equation under appropriate circumstances. (I elaborate on this point in Wallace (2012c)).

${ }^{20}$ For contrasting views on this subject, see (Callender 2004) and (Price 2004). 
to be recovered. ${ }^{21}$ This constraint seems to have the same nature - and the same conceptual difficulties - as occur in classical statistical mechanics, and as discussed in section 5. Indeed, probability in the de Broglie-Bohm theory is often $^{22}$ introduced to students by analogy with classical statistical mechanics.

However, in section 9 I claimed that there is no need for statistical probability in quantum statistical mechanics, and hence no need for it in classical statistical mechanics understood as a classical limit of quantum statistical mechanics. If this correct, probability in deterministic hidden variable theories is sui generis: it may or may not make sense, but it cannot be assumed to make sense just based on the empirical successes of the methods of classical statistical mechanics.

Having said this, hidden-variable theories need not be deterministic, and even some attempts to construct relativistic versions of the de Broglie-Bohm theory do so by introducing stochastic transitions (e.g.,Dürr, Goldstein, Tumulka, and Zanghi 2004). There seem good reasons to think that such stochastic rules are likely to ensure that any initial hidden-variable state is likely to give rise to dynamics that produce appropriate probabilities (Bub (1997) provides a good discussion of this point). If so, the need for an probability condition in hidden variable theories would be obviated.

In any case, neither stochasticity in hidden-variable dynamics nor probabilistic assumptions about hidden-variable initial states suffices to explain the time-asymmetry of the macrodynamics of a hidden-variable theory: that timeasymmetry is encoded in the evolution of the quantum state, which is independent of the hidden variables. Hidden-variable theories need to make some kind of Simplicity assumption about the initial quantum state to recover this time asymmetry. Indeed, in a hidden variable theory with stochastic dynamics, there is no a priori reason why the direction of the stochasticity in the emergent macrodynamics need be aligned with the direction in the hidden-variable dynamics.

Finally, consider the Everett interpretation. Here it is often ${ }^{23}$ claimed that probability is a particular difficulty. But other than conceptual unfamiliarity (which, I can attest, fades with exposure) and intuitions (which we have no reason to regard as truth-tracking in this context), it is not clear why this should be. After all, at a technical level the branching structure in unmodified quantum mechanics has the same structure as in a stochastic dynamical theory: a collection of histories, and a measure defined upon them with the formal structure of a probability measure. The only differences are that (i) in the stochastic case the measure is supposed to be taken as fundamental, whereas in the quantum case it is derived from underlying features of the theory; (ii) in the stochastic case the intended interpretation is that only one history is physically

\footnotetext{
${ }^{21}$ Every problem is an opportunity: Antony Valentini (see Valentini (1996, 2001, 2010), Valentini and Westman (2004), and references therein) has extensively explored the possibility that violation of this constraint could lead to empirically testable deviations from quantum theory.

22 e.g., in Handfield and Wilson's chapter in this volume.

${ }^{23}$ For instance, Lewis (2007b) calls probability the "Achilles heel" of the Everett interpretation
} 
real, whereas in the Everett interpretation, all are (or at any rate, all with a non-vanishing measure). Intuitively (ii) makes a crucial difference, but I am not aware of convincing reasons why these intuitions are to be trusted. ${ }^{24}$

To elaborate: in section 5 I claimed that if a given probability is objective (is 'chance' in Lewis's terminology), it must be either taken as a primitive or analysed in terms of some non-probabilistic facts. But if it is acceptable to identify the measure in a stochastic theory primitively with chance, it is not obviously less acceptable to identify the measure in quantum theory likewise with chance. ${ }^{25}$

And if chance is to be analysed, then that analysis needs to provide both (a) a formal recovery of a probability measure from non-probabilistic facts, and (b) an explanation of why that recovered measure is chance. But (a), though difficult in stochastic theories, is straightforward in quantum theory: probabilities are identified with the mod-squared-amplitudes of the branches, which are categorical magnitudes in quantum theory with non-probabilistic roles to play in the theory's structure and dynamics. (It should not be surprising that (a) is simpler: the difficulty in stochastic mechanics is that the probability measure over a whole space of histories must be recovered from facts about a single history. In Everett-interpreted quantum theory, both the whole space and the measure are emergent properties of the physical Universe.)

As for (b), if the Everett interpretation had nothing to offer here it would not obviously be worse off than in the non-quantum case: in particular, David Papineau claimed some while ago that the inferential and decision-theoretic links between chance and action are just as mysterious in nonquantum physics and I know of no persuasive refutation. But in fact, David Deutsch (1999), Hilary Greaves and Wayne Myrvold (Greaves 2007a, Greaves and Myrvold 2010) and myself have claimed that it is possible to derive some or all of these links from quantum mechanics and from non-question-begging assumptions of decision theory, essentially by exploiting the symmetries of the quantum state (symmetries that are inevitably broken in non-Everettian physics by the fact that one outcome rather than another actually happens). If so, it would effectively amount to a derivation of Lewis's Principle Principle, and thus of Papineau's two links.

My own view, then (developed in extenso in chapters 4-6 of Wallace (2012a)) is that far from probability being a weakness of the Everett interpretation, Everettian quantum mechanics provides new resources to resolve extant problems in the interpretation of probability. (The literature on the subject is large and controversial, though; for dissenting views, see Price (2010), Kent (2010), Albert (2010), Lewis (2005); see also Greaves (2007b) for a general review.)

Whatever the status of probability in the Everett interpretation, though, the status of the time asymmetry of stochastic processes is reasonably clear: since the Everett interpretation is a pure interpretation of unmodified quantum mechanics, its time asymmetry arises from the mechanism discussed in section

\footnotetext{
${ }^{24}$ An alternative approach (which lies beyond the scope of this chapter) exploits the possibility of a connection between the Everettian multiverse and Lewisian modal realism; cf Wilson (2012).

${ }^{25}$ This strategy was advocated in the 1990s by Simon Saunders (Saunders 1995, 1996, 1998).
} 
9: non-probabilistic constraints on the initial quantum state.

\section{Conclusion}

In pre-quantum physics, probability enters either through stochastic mechanics, in which a deterministic evolution equation is replaced by a probabilistic one, or through statistical mechanics, where a probability distribution is placed over states of a classical system with many degrees of freedom. Both moves lead to stochastic dynamics for macrostate of the system, which is by its nature time-asymmetric. In the former case the origin of the asymmetry is the fundamental time asymmetry of the underlying stochastic mechanics; in the latter case, it arises from a particular probabilistic constraint on the system's initial state. In this second case, however, the detailed form of the macrodynamics are independent of the particular initial-state probabilities, provided only that this constraint is satisfied. In both cases, there are significant conceptual and philosophical problems in making sense of the notion of probability that is being used.

In quantum theory, the statistical move plays no particular role: the results of quantum statistical mechanics arise from the quantum dynamics of individual states and do not depend on any additional probabilistic postulate. As a consequence, debates about the nature of classical statistical-mechanical probability are not of direct relevance to our understanding of the actual world as described by contemporary physics. Probability in contemporary physics arises from the probabilistic nature of quantum theory itself, not from any additional posit.

That 'probabilistic nature' depends on how the quantum measurement problem is resolved. According to dynamical-collapse theories, it is a fundamental stochasticity, analogous to pre-quantum stochastic mechanics. According to (deterministic) hidden-variable theories, it is a consequence of a probability distribution over the hidden variables, analogous to pre-quantum statistical mechanics. According to the Everett interpretation, it is something new, not analogous to either; it is controversial whether this means that Everettian probability is more or less well understood than pre-quantum probability.

The direction of time in the probabilistic macrodynamics of quantum theory is also dependent on the resolution of the measurement problem. In dynamical collapse theories, it is a consequence of the fundamental time-asymmetry of the dynamics. In the Everett interpretation, and in hidden-variable theories, it is a consequence of a non-probabilistic constraint on the initial quantum state.

\section{References}

Albert, D. Z. (2000). Time and Chance. Cambridge, MA: Harvard University Press.

Albert, D. Z. (2010). Probability in the Everett picture. In S. Saunders, J. Barrett, A. Kent, and D. Wallace (Eds.), Many Worlds? Everett, Quantum 
Theory and Reality. Oxford: Oxford University Press.

Arntzenius, F. and H. Greaves (2009). Time reversal in classical electromagnetism. British Journal for the Philosophy of Science 60, 557-584.

Ballentine, L. E. (1970). The statistical interpretation of quantum mechanics. Reviews of Modern Physics 42, 358-381.

Ballentine, L. E. (1990). Quantum Mechanics. Englewood Cliffs: Prentice Hall.

Bassi, A. and G. Ghirardi (2003). Dynamical reduction models. Physics Reports 379, 257.

Brown, H. (2010). Reply to Valentini: 'de Broglie-Bohm theory: Many worlds in denial?'. In Many Worlds? Everett, Quantum Theory, and Reality. Oxford: Oxford University Press.

Brown, H. R. and D. Wallace (2005). Solving the measurement problem: de Broglie-Bohm loses out to Everett. Foundations of Physics 35, 517-540.

Bub, J. (1997). Interpreting the Quantum World. Cambridge: Cambridge University Press.

Callender, C. (2004). There is no puzzle about the low-entropy past. In C. Hitchcock (Ed.), Contemporary Debates in Philosophy of Science, Oxford, pp. 240-256. Blackwell.

Colin, S. and W. Struyve (2007). A Dirac sea pilot-wave model for quantum field theory. Journal of Physics A 40, 7309-7342.

Cushing, J. T., A. Fine, and S. Goldstein (Eds.) (1996). Bohmian Mechanics and Quantum Theory: An Appraisal, Dordrecht. Kluwer Academic Publishers.

Deutsch, D. (1999). Quantum theory of probability and decisions. Proceedings of the Royal Society of London A455, 3129-3137.

Dürr, D., S. Goldstein, R. Tumulka, and N. Zanghi (2004). Bohmian mechanics and quantum field theory. Physical Review Letters 93, 090402.

Dürr, D., S. Goldstein, R. Tumulka, and N. Zanghi (2005). Bell-type quantum field theories. Journal of Physics A38, R1.

Earman, J. (2002). What time reversal invariance is and why it matters. International Studies in the Philosophy of Science 16, 245-264.

Frigg, R. (2009). Typicality and the approach to equilibrium in Boltzmannian statistical mechanics. Philosophy of Science 76, 997-1008. Available online at http://philsci-archive.pitt.edu.

Goldstein, S. (2001). Boltzmann's approach to statistical mechanics. In J. Bricmont, D. Dürr, M. Galavotti, F. Petruccione, and N. Zanghi (Eds.), In: Chance in Physics: Foundations and Perspectives, Berlin, pp. 39. Springer. Available online at http://arxiv.org/abs/cond-mat/0105242. 
Goldstein, S. and S. Teufel (2000). Quantum spacetime without observer: ontological clarity and the conceptual foundations of quantum gravity. In C. Callender and N. Huggett (Eds.), Physics meets Philosophy at the Planck Scale, pp. 275-289. Cambridge: Cambridge University Press.

Greaves, H. (2007a). On the Everettian epistemic problem. Studies in the History and Philosophy of Modern Physics 38, 120-152.

Greaves, H. (2007b). Probability in the Everett interpretation. Philosophy Compass 38, 120-152.

Greaves, H. and W. Myrvold (2010). Everett and evidence. In S. Saunders, J. Barrett, A. Kent, and D. Wallace (Eds.), Many Worlds? Everett, Quantum Theory and Reality. Oxford: Oxford University Press.

Halliwell, J. J. (2010). Macroscopic superpositions, decoherent histories and the emergence of hydrodynamic behaviour. In S. Saunders, J. Barrett, A. Kent, and D. Wallace (Eds.), Many Worlds? Everett, Quantum Theory, and Reality. Oxford: Oxford University Press.

Harrigan, N. and R. W. Spekkens (2010). Einstein, incompleteness, and the epistemic view of states. Foundations of Physics 40, 125.

Hartle, J. (2010). Quasiclassical realms. In S. Saunders, J. Barrett, A. Kent, and D. Wallace (Eds.), Many Worlds? Everett, Quantum Theory, and Reality. Oxford: Oxford University Press.

Hawthorne, J. (2010). A metaphysician looks at the Everett Interpretation. See Saunders, Barrett, Kent, and Wallace (2010), pp. 144-153.

Hoefer, C. (2007). The Third Way on Objective Probability: a Skeptic's Guide to Objective Chance. Mind 116, 549-96.

Jaynes, E. (1957a). Information theory and statistical mechanics. Physical Review 106, 620.

Jaynes, E. (1957b). Information theory and statistical mechanics ii. Physical Review 108, 171.

Kent, A. (2010). One world versus many: the inadequacy of Everettian accounts of evolution, probability, and scientific confirmation. In S. Saunders, J. Barrett, A. Kent, and D. Wallace (Eds.), Many Worlds? -Everett, Quantum Theory, and Reality, Oxford. Oxford University Press.

Kochen, S. and E. Specker (1967). The problem of hidden variables in quantum mechanics. Journal of Mathematics and Mechanics 17, 59-87.

Ladyman, J. (2010). Commentary: Reply to hawthorne: Physics before metaphysics. In S. Saunders, J. Barrett, A. Kent, and D. Wallace (Eds.), Many Worlds? Everett, Quantum Theory, and Reality. Oxford: Oxford University Press.

Lebowitz, J. (2007). From time-symmetric microscopic dynamics to timeasymmetric macroscopic behavior: An overview. Available online at http://arxiv.org/abs/0709.0724. 
Lewis, D. (1980). A subjectivist's guide to objective chance. In R. C. Jeffrey (Ed.), Studies in Inductive Logic and Probability, volume II. Berkeley: University of California Press. Reprinted, with postscripts, in David Lewis, Philosophical Papers, Volume II (Oxford University Press, Oxford, 1986); page numbers refer to this version.

Lewis, D. (1986). Philosophical Papers, Vol. II. Oxford: Oxford University Press.

Lewis, D. (1994). Chance and credence: Humean supervenience debugged. Mind 103, 473-90. Reprinted in David Lewis, Papers in Metaphysics and Epistemology (Cambridge: CUP, 1999), pp. 224-247.

Lewis, P. (2007a). How Bohm's theory solves the measurement problem. Philosophy of Science 74, 749-760.

Lewis, P. J. (2005). Probability in Everettian quantum mechanics. Available online at http://phil-sci.pitt.edu.

Lewis, P. J. (2007b). Uncertainty and probability for branching selves. Studies in the History and Philosophy of Modern Physics 38, 1-14.

Loewer, B. (2002). Determinism and chance. Studies in the History and Philosophy of Modern Physics 32, 609-620.

Malament, D. (2004). On the time reversal invariance of classical electromagnetic theory. Studies in the History and Philosophy of Modern Physics 35, $295-315$.

Maudlin, T. (2007). The Metaphysics within Physics. Oxford: Oxford University Press.

Maudlin, T. (2010). Can the world be only wavefunction? In S. Saunders, J. Barrett, A. Kent, and D. Wallace (Eds.), Many Worlds? Everett, Quantum Theory, and Reality. Oxford: Oxford University Press.

Papineau, D. (1996). Many Minds are No Worse than One. British Journal for the Philosophy of Science 47, 233-241.

Price, H. (2004). On the origins of the arrow of time: Why there is still a puzzle about the low-entropy past. In C. Hitchcock (Ed.), Contemporary Debates in Philosophy of Science, Oxford, pp. 219-239. Blackwell.

Price, H. (2010). Probability in the Everett picture. In S. Saunders, J. Barrett, A. Kent, and D. Wallace (Eds.), Many Worlds? Everett, Quantum Theory and Reality. Oxford: Oxford University Press.

Pusey, M. F., J. Barrett, and T. Rudolph (2011). On the reality of the quantum state. Nature Physics 8, 476. arXiv:1111.3328v2.

Saunders, S. (1995). Time, Decoherence and Quantum Mechanics. Synthese 102, 235-266.

Saunders, S. (1996). Time, Quantum Mechanics and Tense. Synthese 10\%, $19-53$. 
Saunders, S. (1998). Time, Quantum Mechanics, and Probability. Synthese 114, 373-404.

Saunders, S., J. Barrett, A. Kent, and D. Wallace (Eds.) (2010). Many Worlds? Everett, Quantum Theory, and Reality, Oxford. Oxford University Press.

Schlosshauer, M. (2007). Decoherence and the Quantum-to-Classical Transition. Berlin: Springer.

Spekkens, R. W. (2007). In defense of the epistemic view of quantum states: a toy theory. Physical Review A 75, 032110.

Struyve, W. and H. Westman (2007). A minimalist pilot-wave model for quantum electrodynamics. Proceedings of the Royal Society of London A 463, $3115-3129$

Tumulka, R. (2006). Collapse and relativity. In A. Bassi, T. Weber, and N. Zanghi (Eds.), Quantum Mechanics: Are There Quantum Jumps? and on the Present Status of Quantum Mechanics, pp. 340. American Institute of Physics Conference Proceedings. Available online at http://arxiv.org/abs/quant-ph/0602208.

Uffink, J. (2010). Irreversibility in stochastic dynamics. In G. Ernst and A. Hütteman (Eds.), Time, Chance and Reduction: Philosophical Aspects of Statistical Mechanics, pp. 180-209. Cambridge: Cambridge University Press.

Valentini, A. (1996). Pilot-wave theory of fields, gravitation and cosmology. See Cushing, Fine, and Goldstein (1996), pp. 45-67.

Valentini, A. (2001). Hidden variables, statistical mechanics and the early universe. In J. Bricmont, D. Dürr, M. C. Galavotti, G. Ghirardi, and F. Petruccione (Eds.), Chance in Physics: Foundations and Perspectives, pp. 165-181. London: Springer. Available online at http://arxiv.org/abs/quant-ph/0104067.

Valentini, A. (2010). De Broglie-Bohm pilot wave theory: Many worlds in denial? In Many Worlds? Everett, Quantum Theory and Reality. Oxford: Oxford University Press.

Valentini, A. and H. Westman (2004). Dynamical origin of quantum probabilities. Available online at http://arxiv.org/abs/quant-ph/0403034.

Wallace, D. (2003). Everett and Structure. Studies in the History and Philosophy of Modern Physics 34, 87-105.

Wallace, D. (2010a). Decoherence and ontology: Or: How I learned to stop worrying and love FAPP. In S. Saunders, J. Barrett, A. Kent, and D. Wallace (Eds.), Many Worlds? Everett, Quantum Theory, and Reality, pp. 53-72. Oxford: Oxford University Press.

Wallace, D. (2010b). The logic of the past hypothesis. Available online at http://users.ox.ac.uk/ mert0130/papers.shtml. 
Wallace, D. (2011). Decoherence and its role in the modern measurement problem. Forthcoming in Philosophical Transactions of the Royal Society $A$; available online at http://arxiv.org/abs/1111.2187.

Wallace, D. (2012a). The Emergent Multiverse: Quantum Theory according to the Everett Interpretation. Oxford: Oxford University Press.

Wallace, D. (2012b). The everett interpretation. In R. Batterman (Ed.), The Oxford Handbook of Philosophy of Physics. Oxford: Oxford University Press.

Wallace, D. (2012c). Statistical mechanics is not statistical. In preparation.

Wallace, D. (2013). Irreversibility, emergence and equilibrium in a quantum universe. In preparation.

Wilson, A. (2012). Objective Probability in Everettian Quantum Mechanics. Forthcoming in British Journal for the Philosophy of Science..

Objective Probability in Everettian Quantum Mechanics

Zurek, W. H. (1991). Decoherence and the transition from quantum to classical. Physics Today 43, 36-44. Revised version available online at http://arxiv.org/abs/quant-ph/0306072. 